\title{
IN VITRO SYNERGISTIC ACTIVITY OF ANTIBIOTIC COMBINATIONS AGAINST BRUCELLA MELITENSIS USING E-TEST METHODOLOGY
}

\author{
Selcuk Kilic ${ }^{1}$; Murat Dizbay²; Kenan Hizel²; Dilek Arman² \\ ${ }^{1}$ Refik Saydam National Hygiene Center, Department of Communicable Diseases Research; ${ }^{2}$ Gazi University Faculty of \\ Medicine, Department of Infections Diseases
}

Submitted: June 21, 2007; Returned to authors for corrections: September 22, 2007; Approved: January 20, 2008.

\begin{abstract}
The treatment of brucellosis is still problematic, because of high rates of treatment failure or relapses. As the microorganism is an intracellular pathogen, treatment requires combined regimens. However, limited existing data on in vitro combinations are avaliable for Brucellae. The aim of this study was to investigate the in vitro efficacy of various traditional and new antibiotic combinations against 16 Brucella melitensis strains. The combination effect of antimicrobial agents was evaluated by E-test synergy method to obtain a fractional inhibitory concentration (FIC) index. Co-Trimoxazole (SXT) and moxifloxocin (MXF) exhibited the lowest MIC, while Rifampin (RIF) had the highest MIC in the study. Combinations with RIF showed the best synergistic activity (100\% of RIF-tetracycline (TET), and $87.5 \%$ of RIF-SXT). Synergistic activity was also detected at seven (43.7\%) of ciprofloxocin (CIP)-SXT, four (25\%) of TET-MXF, and two (12.5\%) of TET-SXT combinations. The combinations that demonstrated additivity were TET-SXT, CIP-SXT and TET-MXF. Antagonism was observed only with the TET-Streptomycin (STR) combination in three strains (18.8\%). Further work including randomized controlled clinical trials is required to fully evaluate the usefulness of these data.
\end{abstract}

Key-words: Brucella melitensis, antibiotics, synergism.

Brucellosis is one of the world's major zoonotic diseases and still remains a significant public health problem mainly in the Mediterranean littoral, Arabian Gulf, Indian subcontinent, in Central Asia, and in Central and South America (10,24).

Because of the intracellular survival in the phagocytic cells of Brucella spp., treatment of brucellosis requires not only combined regimens of antibiotics but agents that have ability to penetrate into macrophages and retain efficacy even in acidic milieu as well $(24,25)$. The World Health Organization (WHO) Expert Committee on Brucellosis recommended treatment with a doxycycline (DOX) and Rifampin (RIF) combination for a 6week course (10). Alternatively, rifampin could be replaced with streptomycin (STR). The high rates of therapeutic failure and relapses, toxicity and side effects of conventional antibiotic regimens have led to the investigation of new drugs and therapeutic schedules restricted largely to the addition of a third drug (usually Co-trimoxazole -SXT) or substitution other aminoglycosides for STR $(25,27)$. On the other hand, controlled clinical trials with other antimicrobials, including SXT, new macrolides, and $\beta$-lactams have shown inferior results or involved few patients for a proper evaluation in this setting (9). Therefore, there is no conclusive evidence on the optimum antibiotic therapy for brucellosis.

Although there are some studies on in vitro susceptibility of Brucella strains to newer and conventional antibiotics, the studies comparing in vitro synergistic activity of well-known traditional combination regimens are very limited $(3,8,11,19,22$, 26,28). The Epsilometer test (E-test), an agar diffusion method, determines not only antimicrobial susceptibility quantitatively, but assesses the synergistic activities of antimicrobial combinations against a wide variety of organisms as well $(6,8$, $18,22,30)$.

*Corresponding Author. Mailing address: Refik Saydam National Hygiene center, Communicable Diseases Research, Cemal Gursel Street no:18 Sihhiye, Ankara. Tel.: +90-312-458 21 69. E-mail: selcuk.kilic@ rshm.gov.tr 
The aim of this study was to assess the activity of conventional and newer antimicrobial combinations using Etest against Brucella melitensis strains.

Sixteen strains of $B$. melitensis were obtained from blood cultures between 2004 and 2005 in Gazi University Hospital in Ankara, Turkey. All of the strains were identified as B. melitensis biovar 3 using the following tests: the requirement of $\mathrm{CO}_{2}$ for growth, production of urease and $\mathrm{H}_{2} \mathrm{~S}$, sensitivity to the dyes basic fuchsin and thionin (at final concentration of 20-40 $\mu \mathrm{g}$ / $\mathrm{ml}$ ), and lysis by the phage Tbilisi and agglutination with monospecific antisera for $\mathrm{A}$ and $\mathrm{M}$ antigens (2). The strains were stored in skim milk at $-80^{\circ} \mathrm{C}$ and twice subcultured before starting the study.

Three Brucella reference strains [Brucella abortus 544 (ATCC 23448), B. melitensis 16M (ATCC 23456), and B. suis 1330 (ATCC 23444)] were used as controls for identification, biotyping and antimicrobial susceptibility testing. In addition to these Brucella reference strains, Escherichia coli ATCC 25922, Staphylococcus aureus ATCC 29213 were also used as control.

Determination of MIC and synergy by E-test methodology was performed in duplicate according to the manufacturer's recommendations. For the evaluation of the MICs of single antimicrobial agents, TET, RIF, SXT, MXF, CIP and STR, E-test was performed on Mueller-Hinton agar (MHA) supplemented with 5\% sheep blood. MHA plates were inoculated with 0.5 McFarland turbidity standard suspensions of each isolate and E-Test strips (AB Biodisk, Solna Sweden) were placed onto each MHA plate. The results were evaluated after 48 hours of incubation in ambient air at $35^{\circ} \mathrm{C}(12)$.

Seven different antibiotic combinations (TET-RIF, TET-STR, TET-SXT, TET-MXF, CIP-STR, CIP-SXT, and RIF-SXT) were evaluated for 16 strains of $B$. melitensis biovar 3 by E-test prediffusion technique to obtain the fractional inhibitory concentration (FIC) index according to manufacturer's recommendations. For testing of drug combinations, after inoculation of the bacterial suspension with turbidities equivalent to $0.5 \mathrm{McF}$ arland onto same culture medium, E-test strips (drug A) were applied and after $1 \mathrm{~h}$ incubation at room temperature the first E-test strip was removed. Afterwards, an E-test strip of second antimicrobial (drug B) was applied onto the imprint of strip A, vertically transposed so MICA and MICB overlap at the same position. E-test method was performed in duplicate for all organisms and combinations. The plates were then incubated at $35^{\circ} \mathrm{C}$ in ambient air and MIC levels of each combination was evaluated after $48 \mathrm{~h}$.

The following formulas were used to calculate the $\Sigma$ FIC (the cumulative fractional inhibitory concentration index): FIC of drug $\mathrm{A}=$ (MIC of drug $\mathrm{A}$ in combination)/(MIC of drug A alone), FIC of drug B = (MIC of drug B in combination)/ (MIC of drug B alone) and $\Sigma$ FIC $=$ FIC of drug A + FIC of drug B.
The results were interpreted according to FIC indexes as follows: synergistic ( $\Sigma \mathrm{FIC}: \leq 0.5$ ), additative ( $\Sigma \mathrm{FIC}:>0.5$ and $\leq 1)$, indifferent ( $\Sigma$ FIC: $>1$ and $\leq 4)$, antagonistic $(\Sigma$ FIC: $>4)$.

The Minimum inhibitory concentrations (MICs) of relevant antibiotics and $\mathrm{MIC}_{50}$ and $\mathrm{MIC}_{90}$ levels defined as the lowest concentration of the antibiotic at which $50 \%$ and $90 \%$ of the isolates inhibited respectively, are shown in Table 1.

Table 1. MIC ranges, $\mathrm{MIC}_{50}$ and $\mathrm{MIC}_{90}$ values of antibiotics against B. melitensis biovar $3(\mathrm{n}=16)$.

\begin{tabular}{cccc}
\hline $\begin{array}{c}\text { Antimicrobial } \\
\text { Agent }\end{array}$ & $\begin{array}{c}\text { MIC ranges } \\
(\mu \mathrm{g} / \mathrm{ml})\end{array}$ & $\begin{array}{c}\mathrm{MIC}_{50} \\
(\mu \mathrm{g} / \mathrm{ml})\end{array}$ & $\begin{array}{c}\mathrm{MIC}_{90} \\
(\mu \mathrm{g} / \mathrm{ml})\end{array}$ \\
\hline SXT & $0.032-0.125$ & 0.064 & 0.094 \\
MXF & $0.032-0.19$ & 0.064 & 0.19 \\
TET & $0.047-0.25$ & 0.094 & 0.25 \\
CIP & $0.064-0.25$ & 0.125 & 0.25 \\
STR & $0.25-0.75$ & 0.50 & 0.75 \\
RIF & $0.75-2$ & 1.5 & 2 \\
\hline
\end{tabular}

SXT: Co-Trimoxazole, MXF: Moxifloxocin, TET: Tetracycline, CIP: Ciproflaxocin, STR: Streptomycin, RIF: Rifampin.

According to the $\mathrm{MIC}_{90}$ values, the most active antibiotic was SXT (MIC $\left.{ }_{90} 0.094 \mu \mathrm{g} / \mathrm{ml}\right)$, followed by MXF $(0.19 \mu \mathrm{g} / \mathrm{ml})$, CIP and TET $(0.25 \mu \mathrm{g} / \mathrm{ml})$, and STR $(0.75 \mu \mathrm{g} / \mathrm{ml})$. RIF had the highest $\mathrm{MIC}_{50}$ and $\mathrm{MIC}_{90}$ values.

The highest rates of synergy were observed with combinations of RIF-TET (100\%) and RIF-SXT $(87.5 \%)$. Synergistic activity was also detected at seven $(43.7 \%$ ) of CIPSXT, four (25\%) of TET-MXF, and two (12.5\%) of TET-SXT combinations. Additive activity was found at nine $(56.2 \%)$ of CIP-SXT, seven $(43.7 \%)$ of TET-SXT and TET-MOX, one (6.2\%) of CIP-STR, and two (12.5\%) of TET-STR combinations.

CIP-STR and TET-STR combinations resulted in indifferent effect against $93.8 \%$ and $68.8 \%$, respectively. Synergy was not seen with TET in combination with STR against any of the strains. The E-test synergy method for the B.melitensis isolates using TET plus STR revealed antagonism in three of $16(18.8 \%)$ (Table 2).

Treatment failures and relapses are major problems in the management of brucellosis $(13,14,20)$. Therapeutic failure is mostly related to pharmacokinetics and pharmacodynamics of antibiotics rather than resistance (25). Relapses usually occur in the first year after the onset of infection, but they are caused by inadequate treatment (inappropriate dosage, short-term administration, and poor patient compliance) in most of cases. In addition, pharmacokinetics and celluler immun status may play significant role in the occurence of relapses $(20,25)$. 
Table 2. Synergy tests results of antibiotic combinations by E-test.

\begin{tabular}{ccccccccccccccc}
\hline \multirow{2}{*}{$\begin{array}{c}\text { Strain } \\
\text { no. }\end{array}$} & \multicolumn{2}{c}{ TET - RIF } & \multicolumn{2}{c}{ TET-STR } & \multicolumn{2}{c}{ RIF-SXT } & \multicolumn{2}{c}{ TET-SXT } & \multicolumn{2}{c}{ CIP-SXT } & \multicolumn{2}{c}{ TET-MXF } & CIP-STR \\
\cline { 2 - 6 } & $\Sigma$ FIC & Activity* & $\Sigma$ FIC & Activity & $\Sigma$ FIC & Activity & $\Sigma$ FIC & Activity & $\Sigma$ FIC Activity & $\Sigma$ FIC & Activity & $\Sigma$ FIC Activity \\
\hline 1 & 0.18 & S & 2.32 & ID & 0.51 & ADD & 1.50 & ID & 0.83 & ADD & 0.40 & S & 1.25 & ID \\
2 & 0.15 & S & 2.08 & ID & 0.35 & S & 0.28 & S & 0.25 & S & 0.22 & S & 1.47 & ID \\
3 & 0.30 & S & 2.73 & ID & 0.20 & S & 0.92 & ADD & 0.40 & S & 1.12 & ID & 1.71 & ID \\
4 & 0.19 & S & 1.57 & ID & 0.17 & S & 1.48 & ID & 0.26 & S & 1.16 & ID & 0.77 & ADD \\
5 & 0.21 & S & 1.70 & ID & 0.29 & S & 1.04 & D & 0.58 & ADD & 1.03 & ID & 2.66 & ID \\
6 & 0.25 & S & 2.18 & ID & 0.13 & S & 1.04 & ID & 0.49 & S & 0.82 & ADD & 2.05 & ID \\
7 & 0.16 & S & 1.70 & ID & 0.25 & S & 0.82 & ADD & 0.53 & ADD & 0.79 & ADD & 2.01 & ID \\
8 & 0.12 & S & 2.12 & ID & 0.39 & S & 0.54 & ADD & 0.59 & ADD & 0.56 & ADD & 1.53 & ID \\
9 & 0.10 & S & 3.04 & ID & 0.20 & S & 0.30 & S & 0.29 & S & 0.24 & S & 1.21 & ID \\
10 & 0.38 & S & 5.50 & AG & 0.29 & S & 0.75 & ADD & 0.60 & ADD & 1.17 & ID & 3.91 & ID \\
11 & 0.28 & S & 0.53 & ADD & 0.23 & S & 1.06 & ID & 0.42 & S & 0.55 & ADD & 1.54 & ID \\
12 & 0.18 & S & 0.62 & ADD & 0.81 & ADD & 1.12 & ID & 0.37 & S & 0.55 & ADD & 1.02 & ID \\
13 & 0.31 & S & 6.05 & AG & 0.38 & S & 0.54 & ADD & 0.58 & ADD & 0.58 & ADD & 2.66 & ID \\
14 & 0.12 & S & 2.18 & ID & 0.35 & S & 0.72 & ADD & 0.51 & ADD & 0.56 & ADD & 1.53 & ID \\
15 & 0.27 & S & 4.06 & AG & 0.39 & S & 2.12 & ID & 0.75 & ADD & 1.18 & ID & 2.97 & ID \\
16 & 0.40 & S & 2.75 & ID & 0.26 & S & 0.58 & ADD & 0.60 & ADD & 0.47 & S & 3.02 & ID \\
\hline
\end{tabular}

TET: Tetracycline; RIF: Rifampin; STR: Streptomycin; SXT: Co-Trimoxazole; CIP: Ciproflaxocin; MXF: Moxifloxocin; S: Synergy; ADD: Additive; ID: Indifference; AG: Antagonism.

In this present study, we detected in vitro susceptibilities of antimicrobials commonly used in brucellosis and also investigated synergistic activity between some old and new antibiotic combinations by using E-test.

Up to now, the standard procedures for in vitro susceptibility testing have not been determined for Brucella species. Currently, methods for MIC determination are described for potential bioterrorism agents including Brucella species by the Clinical Laboratory Standarts Institute (CLSI; formerly the NCCLS). The CLSI proposes the microbroth dilution method using Brucella broth for Brucella spp. The breakpoints used for interpretation as susceptible were as follows: TET/DOX $\leq 1$ $\mu \mathrm{g} / \mathrm{ml}$, SXT $\leq 2 / 38 \mu \mathrm{g} / \mathrm{ml}$, and STR $\leq 8 \mu \mathrm{g} / \mathrm{ml}$ according to the the CLSI interpretive criteria (7). In our study, E-test method was used due to the fact that it was found to be reliable, reproducible, less labor-intensive, less time-consuming, and more practical than the broth micro dilution method $(12,17,22,29)$.

Three methods to detect in vitro synergy have been described: the time-kill assay (TKA), checkerboard titration technique, and E-test. However, neither is applied in routine microbiology laboratories. Whilst most synergy studies have been performed using checkerboard or TKA, these methods are too time consuming and technically challenging for routine clinical testing. Therefore, we preferred E-test method on grounds of simplicity and availability to routine clinical laboratories $(18,22,23)$.
Since the standard protocols for antimicrobial susceptibility and the breakpoint values have not been defined up to now, the CLSI interpretive criteria for slow growing bacteria (Haemophilus) have been taken into consideration in order to evaluate the results of MICs determination in the literature $(4,17,29)$. The MIC values of TET, STR, SXT, CIP, MXF interpreted according to the CLSI criteria for slow growing bacteria and potential bioterrorism agents have shown ranges below the breakpoints for sensitivity determination (7).

The MIC values of RIF range $0.75-2 \mu \mathrm{g} / \mathrm{ml}$ at levels below the breakpoints for resistance determination according to the CLSI interpretive criteria, however, MIC value of 12 of our isolates of the total $16(75 \%)$ were found to be $\geq 1 \mu \mathrm{g} / \mathrm{ml}$ and these strains may be characterized as intermediately resistant by CLSI definition. MIC values of RIF in previous studies were reported to range from 0.047 to $4 \mu \mathrm{g} / \mathrm{ml}$, values confirmed again by our findings $(4,5,11,17,19,28,29)$.

SXT demonstrated the lowest $\mathrm{MIC}_{50}$ and $\mathrm{MIC}_{90}$ in our study and these were significantly lower than those previously observed in Turkey $(4,5,17)$. Although higher MIC values of SXT have been determined in Turkey with MICs ranging from 0.047 to $3.0 \mu \mathrm{g} / \mathrm{ml}$, in vitro SXT resistance rate was reported as low as $2 \%$ (4). However, significant rates of SXT resistance have been reported in the world $(15,21)$.

MIC values of tetracycline were low, corresponding to in vitro susceptibility of all isolates, which is consistent with 
previous reports $(4,5,17,26,29,31)$. In vitro susceptibility to STR was also found to be in the range described previously $(3,22,29)$. However, the MICs of STR were significantly lower than those reported by Garcia-Rodriguez et al. (11), Trujillano-Martin et al. (28), Lopez-Merino et al. (19) and Rubenstein et al. (26). These higher MIC values for STR may be related to in vitro testing method used in these studies.

CIP and MXF have shown good in vitro activity to Brucella strains which were comparable to that of tetracycline in our study. In previous studies from Turkey $(4,5,16,17)$, similarly, fluoroquinolones were found very active drugs against B.melitensis in vitro except that a study in which higher $\mathrm{MIC}_{90}$ levels of CIP $(2 \mu \mathrm{g} / \mathrm{ml})$ and MXF $(8 \mu \mathrm{g} / \mathrm{ml})$ were determined using agar dilution method (31).

There is very limited data about in vitro synergistic activity of Brucella strains in the literature so far though several antibiotic combinations have been used in the treatment of brucellosis such as TET-STR, DOX-STR, DOX-RIF, RIF-SXT for many years. We determined in vitro synergistic activity of seven new and old antibiotic combinations in this study. Although RIF had the highest MIC values, the best synergistic activity was observed in $100 \%$ and $87.5 \%$ of the TET-RIF and RIF-SXT combinations, respectively. In contrast, synergistic activity was not detected in the combinations with STR (TET-STR and CIP-STR). Moreover, antagonistic effect was seen only in TET-STR combination $(12.5 \%)$ which it is recommended for the classical treatment of brucellosis. SXT in combination with TET and CIP led to reduced significant MICs with most strains but this was not synergistic ( $\mathrm{FICI}=0.54-0.92$ and FICI $=0.53-0.83$, respectively). In contrast to our findings, a study comparing checkerboard with E-test assays on 16 B.melitensis isolates did not demonstrate antagonism between STR with DOX (22). In the study by Orhan et al. (22) testing for synergy between RIF, DOX, STR and SXT, synergy with RIF-DOX, STR-DOX, RIF-SXT, and SXT-DOX combinations were determined by E-test method, as $93.7 \%$, $68.7 \%, 37.5 \%$ and $37.5 \%$ respectively. The reasons for the discrepancies between two studies may be explained by either different E-test testing procedure used to assess the synergy or regional differences in susceptibilities of B.melitensis strains.

As synergy testing methods are not yet standardized for reproducibility and interpretation, it is extremely difficult to compare these methods' results from different studies. Three Etest methods have been recently described: (i) a prediffusion technique used in this study; (ii) which Etest strips were placed side by side; and (iii) a second Etest strip was placed on the agar in a cross formation, with a $90^{\circ}$ angle at the intersection between the scales at the respective MICs for the organism. Only the prediffusion method was reproducible, and has shown higher aggrement rates with TKA than those of other E-test synergy methods (30). The third E-test synergy testing method was applied by Orhan et al. and antagonism was difficult to detect with their method as previously reported $(6,30)$. In addition, controversial results for classical combinations used in the therapy were also obtained from killing studies. In the study by Al-Dahouk et al. (1) testing for killing activity between DOX-RIF and DOX-STR, it was shown that the combination of DOX with STR did not exhibit killing activity. This finding is in agreement with our result in which no synergistic effect of TET plus STR was observed.

Despite the fact that quinolones are not recommended in first-line therapy for brucellosis, we detected $43.7 \%$ and $25 \%$ synergistic activity and $56.2 \%$ and $43.7 \%$ additive activity in CIP-SXT and TET-MXF combinations, respectively. These results may lead one to consider to use quinolones as an alternative choice if toxicity occurs in the classical combinations or as part of a second-line regimen in patients who fail to respond or develop disease relapse after therapy.

In conclusion, though Etest synergy method is relatively new and the use of the Etest strips for synergy has yet to be standardized, prediffusion technique has the potential to be a useful screening test for the determination of synergy due to easier to perform and time-efficient. The combinations with RIFDOX and RIF-SXT appeared to be most effective (synergy 100\% and $87.5 \%$, respectively) by E-test prediffusion technique. Antagonistic activity was only seen in TET-STR combination, which it is commonly used in treatment of brucellosis. Although clinical association with these results are yet to be determined, it can be postulated that, other combinations especially with RIF or quinolones could be replaced in treatment failure or late response to the treatment with TET-STR. Considering the fact that in vitro antimicrobial susceptibility does not always predict clinical efficacy, in vivo studies and controlled clinical trials are warranted to confirm these considerations.

\section{RESUMO}

\section{Atividade sinergística in vitro de combinações de antibióticos contra Brucella melitensis através da metodologia E-test}

O tratamento da brucelose é problemático devido à alta frequiência de tratamentos mal sucedidos e recidivas. Por tratarse de um patógeno intracelular, o tratamento requer procedimentos combinados. Entretanto, existem poucos dados sobre combinações in vitro para Brucellae. O objetivo deste trabalho foi investigar a eficiência de vários tratamentos tradicionais e novas combinações de antibióticos contra 16 isolados de Brucella melitensis. O efeito combinado foi avaliado através do método do E-test para obtenção do FIC (índice de concentração inibitória fracional). Co-trimoxazol (SXT) e moxifloxocina (MXF) apresentaram o MIC mais baixo, enquanto rifampicina (RIF) apresentou o MIC mais alto. Combinações com RIF mostraram a melhor atividade sinergística (100\% para RIF-tetraciclina e 87,5\% para RIF-SXT). Atividade sinergística 
foi também detectada para sete $(43,7 \%)$ combinações de ciprofloxacina (CIP-STX), quatro (25\%) de TET-MXF e duas $(12,5 \%)$ de TET-SXT. As combinações que apresentaram efeito aditivo foram TET-SXT, CIP-SXT e TET-MXF. Antagonismo foi observado somente para a combinação TET-estreptomicina (STR) em três isolados $(18,8 \%)$. Mais pesquisas utilizando ensaios clínicos randomizados controlados são necessárias para avaliar a utilidade desses dados.

Palavras-chave: Brucella melitensis, antibióticos, sinergismo

\section{REFERENCES}

1. Al Dahouk, S.; Hagen, R.M.; Nockler, K.; Tomaso, H.; Wittig, M.; Scholz, H.C.; Vergnaud, G.; Neubauer, H. (2005). Failure of a shortterm antibiotic therapy for human brucellosis using ciprofloxacin. A study on in vitro susceptibility of Brucella strains. Chemotherapy, 6, 352-356.

2. Alton, G.G.; Jones, L.M.; Angus, R.D.; Verger, J.M. (1988). Techniques for the Brucellosis Laboratory. Paris: Institut National de la recherche Agronomique (INRA), p. 34-61.

3. Ariza, J.; Bocsh, J.; Gudiol, F.; Linares, J.; Fernandez, V.P.; Martin, R. (1986). Relevance of in vitro antimicrobial susceptibility of Brucella melitensis to relapse rate in human brucellosis. Antimicrob. Agents Chemoter, 30, 958-960.

4. Baykam, N.; Esener, H.; Ergonul, O.; Eren, S.; Celikbas, A.K., Dokuzoguz, B. (2004). In vitro antimicrobial susceptibility of Brucella species. Int. J. Antimicrob. Agents, 23, 405-407.

5. Bodur, H.; Balaban, N.; Aksaray, S.; Yetener, V.; Akinci, E.; Coplan, A.; Erbay, A. (2003). Biotypes and antimicrobial susceptibilities of Brucella isolates. Scand. J. Infect. Dis., 35, 337-338.

6. Bonapace, C.R.; White, R.L.; Friedrich, L.V.; Bosso, J.A. (2000). Evaluation of antibiotic synergy against Acinetobacter baumannii: a comparison with Etest, time-kill, and checkerboard methods. Diagn. Microbiol. Infect. Dis., 1, 43-50.

7. Clinical and Laboratory Standards Institute (2006). Performance standards for antimicrobial susceptibility testing; Sixteenth informational supplement. CLSI document M 100-S16, Wayne, PA; USA.

8. Dizbay, M.; Kilic, S.; Hizel, K.; Arman, D. (2007). Tigecycline: its potential for treatment of brucellosis. Scand. J. Infect. Dis., 39 (5), 432-4.

9. Falagas, M.E.; Bliziotis, I.A. (2006). Quinolones for Treatment of Human Brucellosis: Critical Review of the Evidence from Microbiological and Clinical Studies. Antimicrob. Agents Chemother. $50,22-33$.

10. Food and Agriculture Organization-World Health Organization. Joint FAO/WHO Expert Committee on Brucellosis (sixth report) (1986) WHO Technical Report Series No. 740. Geneva, World Health Organisation, p. 56-57.

11. Garcia-Rodrigez, J.A.; Munoz Bellidio, J.L.; Fresnadillo, J.M.; Trujillano, I. (1993). In vitro activities of new Macrolides and Rifepentine against Brucella Spp. Antimicrob. Agents Chemother. 37, 911-913.

12. Gur, D.; Kocagoz, S.; Akova, M. (1999). Comparison of E-test to microdilution for determining in vitro activities of antibiotics against Brucella melitensis. Antimicrob. Agents Chemother, 43, 2337.

13. Hall, W.H. (1991). Modern chemotherapy for brucellosis in humans. Rev. Infect. Dis., 3, 1060-1099.
14. King, A. (2001). Recommendations for susceptibility tests on fastidious organisms and those requiring special handling. $J$. Antimicrob. Chemother, 48 (Suppl S1), 77-80.

15. Kinsara, A.; Al-Mowallad, A.; Osaba, A.O. (1999). Increasing resistance of Brucella to Co-Trimoxazole. Antimicrob. Agents Chemother. 6,1531 .

16. Kocagoz, S.; Akova, M.; Altun, B.; Gur, D.; Hascelik, G. (2002). In vitro activities of new quinolones against Brucella melitensis isolated in a tertiary-care hospital in Turkey. Clin. Microbiol. Infect., 8 , 240-242.

17. Kose, S.; Kilic, S.; Ozbel, Y. (2005). Identification of Brucella species isolated from proven Brucellosis Patient in Izmir, Turkey. J. Basic Microbiol., 45, 323-327.

18. Lewis, R.E.; Diekema, D.J.; Messer, S.A.; Pfaller, M.A.; Klepser, M.E. (2002). Comparison of Etest, chequerboard dilution and timekill studies for the detection of synergy or antagonism between antifungal agents tested against Candida species. Antimicrob. Agents Chemother., 49, 345-351.

19. Lopez-Merino, A.; Contreras-Rodriguez, A.; Migranas-Ortiz, R.; Orrantia-Gradin, R.; Hernandez-Oliva, G.M.; Gutierrez-Rubio, A.T.; Cardenosa, O. (2004). Susceptibility of Mexican Brucella isolates to moxifloxacin, ciprofloxacin and other antimicrobials used in the treatment of human brucellosis. Scand. J. Infect. Dis., 36, 636-638.

20. Madkour, M.M. (2001). Treatment. In: Madkour MM, (ed): Madkour's Brucellosis. Berlin Heidelberg New York: Springer-Verlag, p. 241-261.

21. Memish, Z.; Mah, M.W.; Al Mahmoud, S.; Al Shaalan, M.; Khan, M.Y. (2000). Brucella bacteraemia: Clinical and laboratory observations in 160 Patients. J. Infect., 1, 59-63.

22. Orhan, G.; Bayram, A.; Zer, Y.; Balci, I. (2005). Synergy tests by Etest and checkerboard methods of antimicrobial combinations against Brucella melitensis J. Clin. Microbiol., 43, 140-143.

23. Pankey, G.A.; Ashcraft, D. (2005). In Vitro Synergy of Ciprofloxacin and Gatifloxacin against Ciprofloxacin-Resistant Pseudomonas aeruginosa. Antimicrob. Agents Chemother., 7, 2959-2964.

24. Pappas, G.; Christou, L.; Akritidis, N.; Bosilkovski, M.; Tsianos, E. (2005). Brucellosis. N. Engl. J. Med., 352, 2325-2336.

25. Pappas, G.; Solera, J.; Akritidis, N.; Tsianos, E. (2005). New approaches to the antibiotic treatment of brucellosis. Int. J. Antimicrob. Agents, 26, 101-105.

26. Rubinstein, E.R.; Lang, R.; Shasha, B.; Hagar, B.; Diamanstein, L.; Joseph, G.; Anderson, M.; Harrison, K. (1991). In vitro susceptibility of Brucella melitensis to antibiotics. Antimicrob. Agents Chemother, 35, 1925-1927.

27. Solera, J.; Martinez-Alfaro, E.; Espinosa, A. (1997). Recognition and optimum treatment of brucellosis. Drugs, 53: 245-256.

28. Trujillano-Martin, I.; Garcia Sanchez, E.; Martinez, I.M.; Fresnadillo, M.J.; Garcia Sanchez, J.E.; Garcia-Rodrigez, J.A. (1999). In vitro activities of six new Fluoroquinoles against Brucella melitensis. Antimicrob. Agents Chemoter., 1, 194-195.

29. Turkmani, A.; Ioannidis, A.; Christidou, A.; Psaroulaki, A.; Loukaides, F.; Tselentis, Y. (2006). In vitro susceptibilities of Brucella melitensis isolates to eleven antibiotics. Annals Clin. Microbiol. Antimicrob., $5,24-29$.

30. White, R.L.; Burgess, D.S.; Manduru, M.; Bosso, J.A. (1996) Comparison of three different in vitro methods of detecting synergy: time-kill, checkerboard, and E-Test. Antimicrob. Agents Chemother. 8, 1914-1918

31. Yamazhan, T.; Aydemir, S.; Tunger, A.; Serter, D.; Gokengin, D. (2005). In vitro activities of various antimicrobials against Brucella melitensis strains in the Aegean Region in Turkey. Med. Princ. Pract. 14, 413-416. 